\title{
Stochastic stability of the loaded stiff rod
}

\section{H. ROOZEN}

Centre for Mathematics and Computer Science, P.O. Box 4079, 1009 AB Amsterdam, The Netherlands Present address: University of Amsterdam, Faculty of Mathematics and Computer Science, Plantage Muidergracht 24, 1018 TV Amsterdam, The Netherlands

Received 1 December 1988; accepted in revised form 17 April 1989

\begin{abstract}
A stiff rod, held in a vertical position by an elastic hinge, is subjected to a load consisting of a deterministic and a small stochastic component, both acting in fixed directions. The rod is slightly damped and carries out small oscillations around an equilibrium position. Above a critical energy level, the mechanical system of which the rod forms a part, may get damaged. At some time, an accumulation of stochastic effects can lead to an excess of this critical energy level. In this paper we derive various statistical expressions related to the time needed to reach the critical energy level. These expressions can be adopted as a measure of the reliability of the mechanical system.
\end{abstract}

\section{Introduction}

In their paper [7], Katz and Schuss considered the reliability of elastic structures with random loads. The reliability of such structures was treated as an exit problem in the theory of stochastic dynamical systems. Starting with the simple pendulum, a sequence of models has been considered with increasing complexity, viz. the double pendulum, the $n$-fold pendulum and the elastic continuous column. It has been shown that the exit behaviour of these more complex pendula is essentially the same as that of the simple pendulum. In this paper we will study the simple pendulum (or stiff rod) into more detail than was done by Katz and Schuss. In contrast to their approach, in which both the deterministic and stochastic loads to the simple pendulum were applied vertically, we will allow these loads to act independently from each other, in arbitrary fixed directions.

In Section 2 we will give a description of the stiff rod model and derive the stochastic equation in dimensionless form. The deterministic load is of order $\mathrm{O}\left(\varepsilon^{0}\right)$, the stochastic load has intensity of order $\mathrm{O}(\varepsilon)$, and the damping is of order $\mathrm{O}(\varepsilon)$, where $0<\varepsilon \ll 1$ is a small parameter. In Section 3 we will derive the backward equation, valid on the time scale of order $\mathrm{O}\left(\varepsilon^{-1}\right)$, which is needed in the study of the problem of exit from an energy interval. This interval, bounded below by zero and above by a critical energy, is scaled to the unit interval. In the Sections 4,5 and 6 we will distinguish three cases, according to the magnitude of the angles under which the deterministic and the stochastic loads act. The regular case is treated in Section 4, while Sections 5 and 6 treat two special cases. For each of these cases, we will derive expressions for the expected exit time from the unit interval. For some special cases the exit-time density, the corresponding cumulants and some other related expressions will be derived as well. In Section 7 we give some of the results in dimensional form. The discussion in Section 8 is directed towards some practical aspects of the results obtained. 


\section{The model}

An unloaded stiff rod of length $l$, with mass $m$ at a distance $l^{\prime}$ from the hinge $O$, and spring constant $\mu$ at $O$. carries out small oscillations around the equilibrium position $\varphi=0$. Next a deterministic load $P_{d}$ is applied to the rod, acting under the fixed angle $\varphi_{d}$, as indicated in Fig. 1. The potential energy due to $P_{d}$ is given by

$$
-P_{d} l\left[1-\cos \left(\varphi-\varphi_{d}\right)\right] \approx-P_{d} l\left(1-\cos \varphi_{d}-\varphi \sin \varphi_{d}+\frac{\varphi^{2}}{2} \cos \varphi_{d}\right) \text {. }
$$

where we assumed that $|\varphi|$ is small so that

$$
\sin \varphi \approx \varphi, \quad \cos \varphi \approx 1-\varphi^{2} / 2
$$

The potential energy due to the spring property of the hinge equals

$$
\frac{1}{2} \mu \varphi^{2} l^{2}
$$

Differentiating the total potential energy, which is obtained by adding (2.1) and (2.3), with respect to $\varphi$ and equating this to zero we obtain the new equilibrium position $\varphi_{e}$ :

$$
\varphi_{e}=\frac{-P_{d} \sin \varphi_{d}}{\mu l-P_{d} \cos \varphi_{d}} .
$$

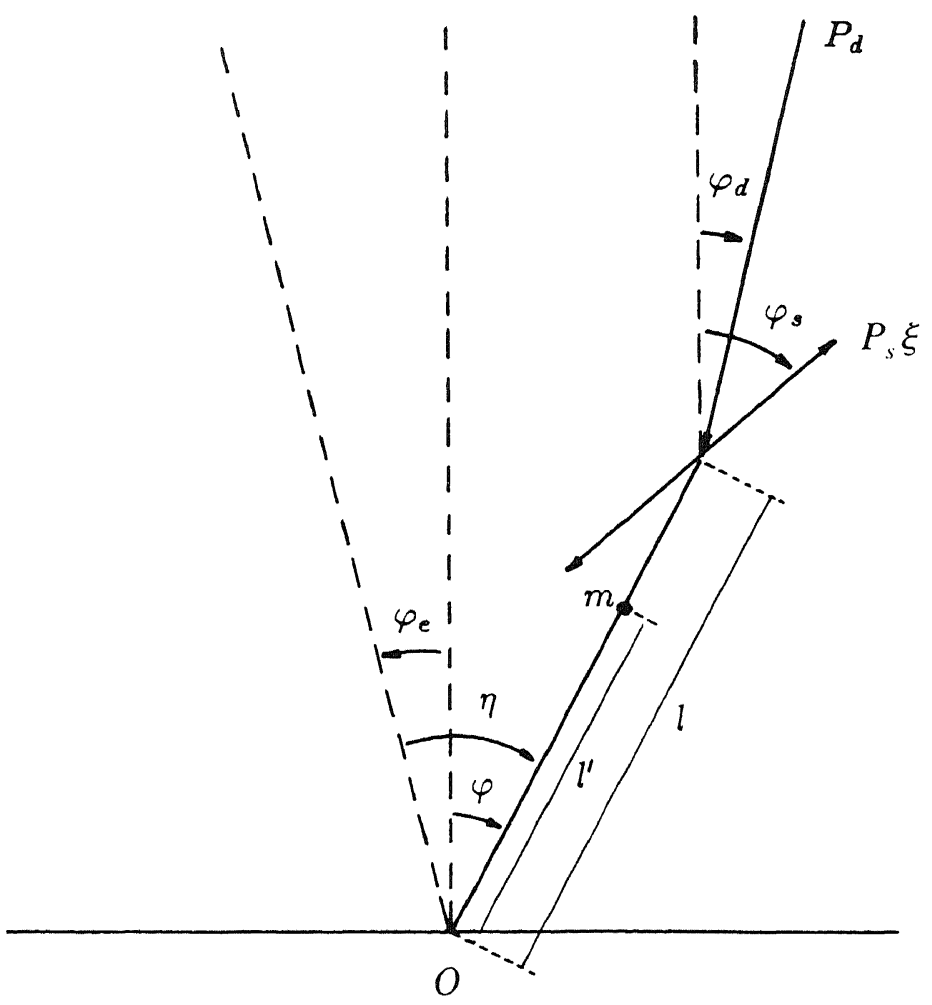

Fig. 1. The loaded stiff rod. 
It is assumed that $\mu l \gg\left|P_{d}\right|$, so that $\left|\varphi_{f}\right|$ is small, in agreement with (2.2). The arbitrary constant that may be added to the potential energy is chosen such that the total potential energy equals zero at $\varphi_{t}$. The kinetic energy of the rod is given by

$$
T=\frac{1}{2} m l^{\prime 2}\left(\frac{\mathrm{d} \varphi}{\mathrm{d} t}\right)^{2}
$$

The Lagrange equation of motion reads

$$
m \frac{l^{\prime 2}}{l^{2}} \frac{\mathrm{d}^{2} z}{\mathrm{~d} t^{2}}+\mu z-\left(\frac{z}{l} \cos \varphi_{d}-\sin \varphi_{d}\right) P_{d}=0 . \quad z=1 \varphi .
$$

To describe a more realistic system, we extend the model with a damping term and a stochastic load component:

$$
m \frac{l^{\prime 2}}{l^{2}} \frac{\mathrm{d}^{2} z}{\mathrm{~d} t^{2}}+\alpha \frac{\mathrm{d} z}{\mathrm{~d} t}+\mu z-\left(\frac{z}{l} \cos \varphi_{d}-\sin \varphi_{d}\right) P_{d}=\left(\frac{z}{l} \cos \varphi_{l}-\sin \varphi_{l}\right) \xi(\gamma t) P_{\mathrm{l}} .
$$

Here $\xi($.$) is a Gaussian white noise process and \gamma$ denotes a constant frequency (an appropriate choice is the unit frequency), so that $\gamma t$ is a dimensionless quantity. $P_{s}$ has the dimension of a load. Note that the stochastic load component is zero for $\varphi=\varphi_{\text {s }}$ (which is possible only if $\varphi$, is small). Here $\varphi$, is the fixed angle under which the stochastic load acts. The damping constant is denoted by $\alpha$. With the abbreviations

$$
\tilde{m}=m \frac{l^{\prime 2}}{l^{2}}, \quad \tilde{\mu}=\mu-\frac{P_{d l}}{l} \cos \varphi_{l l} .
$$

and the change of variable from $z$ to $\eta$ given by

$$
z=\eta-\frac{P_{d} \sin \varphi_{d}}{\tilde{\mu}}
$$

where $\eta$ is the deviation from the equilibrium position (2.4), we have

$$
\tilde{m} \frac{\mathrm{d}^{2} \eta}{\mathrm{d} t^{2}}+\alpha \frac{\mathrm{d} \eta}{\mathrm{d} t}+\tilde{\mu} \eta=\left(\frac{\cos \varphi_{s}}{l} \eta-\frac{P_{d} \sin \varphi_{d} \cos \varphi_{s}}{\tilde{\mu} l}-\sin \varphi_{s}\right) \xi(\gamma t) P_{s} .
$$

Suppose the rod is part of a mechanical structure, that functions well as long as the energy of the rod is below a critical value $R^{2}$ (recall that the energy of the rod takes its minimum value zero when it is at rest at the equilibrium position $\varphi^{\prime}$ determined by the deterministic load). With the transformations

$$
\begin{array}{ll}
\eta^{*}=\eta \sqrt{\tilde{\mu}} / R, & t^{*}=t \sqrt{\tilde{\mu} / \tilde{m}}, \quad P_{d}^{*}=P_{d} / R \sqrt{\tilde{\mu}}, \\
P_{s}^{*}=P_{s} / R \sqrt{\tilde{\mu}}, & \gamma^{*}=\gamma \sqrt{\tilde{m} / \tilde{\mu}},
\end{array}
$$

and the white-noise scaling property

$$
\xi(\gamma t)=\xi\left(\gamma^{*} t^{*}\right)=\frac{1}{\sqrt{\gamma^{*}}} \xi\left(t^{*}\right)
$$


we obtain the equation of motion in dimensionless variables (denoted by ${ }^{*}$ ):

$$
\frac{\mathrm{d}^{2} \eta^{*}}{\mathrm{~d} t^{* 2}}+\frac{\alpha}{\sqrt{\tilde{m} \tilde{\mu}}} \frac{\mathrm{d} \eta^{*}}{\mathrm{~d} t^{*}}+\eta^{*}=\frac{P_{s}^{*}}{\sqrt{\gamma^{*}}}\left(\frac{R \cos \varphi_{s}}{l \sqrt{\tilde{\mu}}}\left(\eta^{*}-P_{d}^{*} \sin \varphi_{d}\right)-\sin \varphi_{s}\right) \xi\left(t^{*}\right) .
$$

We make the following assumptions on the magnitude of the various terms:

$$
\frac{P_{s}^{*}}{\sqrt{\gamma^{*}}}=\sqrt{\varepsilon}, \quad \frac{R \cos \varphi_{s}}{l \sqrt{\tilde{\mu}}}=k_{1}, \quad k_{1} P_{d}^{*} \sin \varphi_{d}+\sin \varphi_{s}=k_{0}, \quad \frac{\alpha}{\sqrt{\tilde{m} \tilde{\mu}}}=\varepsilon k
$$

in which $0<\varepsilon \ll 1$ and $k, k_{0}, k_{1}$ are $\mathrm{O}\left(\varepsilon^{0}\right)$ constants. Equation (2.12) becomes

$$
\ddot{\eta}+\varepsilon k \dot{\eta}+\eta=\sqrt{\varepsilon}\left(k_{1} \eta-k_{0}\right) \xi
$$

where we suppressed the ${ }^{*}$ of $\eta^{*}$ and the dot denotes differentiation with respect to $t^{*}$. Equation (2.14) describes an $\mathrm{O}(\varepsilon)$ damped oscillation of the stiff rod from a load consisting of a deterministic part of $\mathrm{O}\left(\varepsilon^{(1)}\right)$ and a stochastic part with intensity of $\mathrm{O}(\varepsilon)$. We note that $\varphi_{d}$ and $\varphi_{s}$ are arbitrary fixed angles. Equation (2.14) can be written as the system:

$$
\begin{aligned}
& \frac{\mathrm{d} \eta}{\mathrm{d} t^{*}}=\dot{\eta} \\
& \frac{\mathrm{d} \dot{\eta}}{\mathrm{d} t^{*}}=-(\varepsilon k \dot{\eta}+\eta)+\sqrt{\varepsilon}\left(k_{1} \eta-k_{0}\right) \xi .
\end{aligned}
$$

\section{The backward equation}

The undisturbed $(\varepsilon=0)$ system $(2.15)$ is an undamped oscillator, whose dynamics are described by closed trajectories around the origin in the $(\eta, \dot{\eta})$-phase plane. Each trajectory corresponds to an energy level. The energy is larger for orbits farther away from the origin. The effect of a nonzero $\varepsilon$ is that the trajectories tend to spiral inwards to approach the origin (as a consequence of damping) and exhibit stochastic fluctuations in the $\dot{\eta}$-direction. The backward Kolmogorov equation corresponding to (2.15) reads [10, Ch. 5] or [4, Ch. 4]:

$$
\frac{\partial u}{\partial t^{*}}=\dot{\eta} \frac{\partial u}{\partial \eta}-(\varepsilon k \dot{\eta}+\eta) \frac{\partial u}{\partial \dot{\eta}}+\frac{\varepsilon}{2}\left(k_{1} \eta-k_{0}\right)^{2} \frac{\partial^{2} u}{\partial \dot{\eta}^{2}}
$$

We will use this equation with the function $u\left(\eta, \dot{\eta}, t^{*}\right)$ and with various other functions instead of $u$, which will be defined later on. The remainder of this section applies to all these functions. Equation (3.1) is studied asymptotically for small $\varepsilon$ on a time scale of order $\varepsilon^{-1}$. With

$$
t^{*}=\tilde{t} / \varepsilon, \quad u=u^{0}+\varepsilon u^{1}+\cdots,
$$

and the transformation $(\eta, \dot{\eta}) \rightarrow(r, \theta)$ defined by

$$
\eta=\sqrt{2} r \cos \theta, \quad \dot{\eta}=\sqrt{2} r \sin \theta,
$$


we obtain to leading order in $\varepsilon$ :

$$
\frac{\partial u^{0}}{\partial \theta}=0
$$

implying $u^{\prime \prime}=u^{0}(r, \tilde{t})$. Note that $r^{2}$ is the dimensionless energy of the undisturbed $(\varepsilon=0)$ system, $r \in[0,1]$ and $r^{2}=1$ corresponds to the critical energy.

To the next order in $\varepsilon$ an equation is obtained in terms of $u^{0}$ and $u^{1}$. Terms with $u^{1}$ vanish by integration of this equation with respect to $\theta$ from 0 to $2 \pi$ and the additional assumption that $u^{1}$ is periodic in $\theta$ with period $2 \pi$. The resulting equation for $u^{0}$ reads

$$
\frac{\partial u^{0}}{\partial \tilde{t}}=\left(\frac{a_{0}}{r}+a_{2} r\right) \frac{\partial u^{0}}{\partial r}+\left(a_{0}+a_{1} r^{2}\right) \frac{\partial^{2} u^{0}}{\partial r^{2}},
$$

with

$$
a_{0}=\frac{1}{8} k_{0}^{2}, \quad a_{1}=\frac{1}{16} k_{1}^{2}, \quad a_{2}=\frac{3}{16} k_{1}^{2}-\frac{1}{2} k .
$$

Thus, the description to this order in $\varepsilon$ includes damping and stochastic effects. If, as a consequence of the latter effect, the critical energy $r^{2}=1$ is reached in finite time with probability one, starting from $r \in[0,1]$, the rod is stochastically unstable [10]. In that case, the mean exit time from the unit interval at 1 is a measure for the stability (reliability) of the rod (and thus an index of reliability). Below we will discuss this problem of exit from the unit interval. Several cases are distinguished, according to whether $k_{0}, k_{1}$ are equal or unequal to zero.

\section{The regular case}

Let the regular case be defined by $k_{0} \neq 0, k_{1} \neq 0$. This case occurs, in general, when $k_{0}$ and $k_{1}$ are chosen arbitrarily. In this case $a_{0}>0, a_{1}>0$. The boundary $r=0$ of the unit interval is classified as an entrance boundary, see Feller [3], meaning that $r=0$ cannot be reached in finite time from the interior of the interval, and the interior can be reached in finite time from $r=0$. At $r=1$ an absorbing boundary [4] is adopted. It can be reached in finite time from the interior of the interval. On reaching this boundary, absorption occurs, so that the interval cannot be entered again. Thus, exit from the unit interval can take place only at $r=1$. Let $u_{s}(r)$ be the probability of exit at $r=1$, starting from a point $r \in[0,1]$. Its leading-order part $u_{s}^{(0}(r)$ in an expansion in powers of $\varepsilon$ satisfies the stationary backward equation (3.5a) with the boundary condition $u_{s}^{0}(1)=1$. We find $u_{s}(r) \sim u_{s}^{0}(r) \equiv 1$ as the only relevant (i.e. finite) solution. There is no freedom to specify an arbitrary boundary condition at $r=0$. We conclude that if we start somewhere on the interval $[0,1]$, exit at $r=1$ will occur with probability one, so that the oscillator is stochastically unstable. Next we consider the expected exit time $T(r)$, starting from a point $r$. Similar to the time scaling (3.2a) we put $T=\tilde{T} / \varepsilon$ and similar to the expansion $(3.2 b)$ of $u$ we put $\tilde{T}=\tilde{T}^{0}+\varepsilon \tilde{T}^{1}+\cdots$, so that $T=\tilde{T}^{(1)} / \varepsilon+\tilde{T}^{1}+\cdots$. An approximation for $T$ is found by solving the Dynkin equation [10, p. 118]

$$
-1=\left(\frac{a_{0}}{r}+a_{2} r\right) \frac{\partial \tilde{T}^{0}}{\partial r}+\left(a_{0}+a_{1} r^{2}\right) \frac{\partial^{2} \tilde{T}^{0}}{\partial r^{2}},
$$


with the conditions

$$
\tilde{T}^{0}(0) \text { is finite, } \quad \tilde{T}^{0}(1)=0 .
$$

We find

$$
T(r) \sim \frac{1}{\varepsilon\left(a_{1}-a_{2}\right)} \int_{r}^{1}\left[\left(\frac{a_{1}}{a_{0}} s^{2}+1\right)^{-a_{2} / 2 a_{1}+1 / 2}-1\right] \frac{1}{s} \mathrm{~d} s, \quad\left(a_{2} \neq a_{1}\right) .
$$

Only if the exponent in the integrand is a simple rational number, the integral reduces to a simple expression. If the exponent is equal to a positive integer, the binomial expansion can be used to evaluate the integral. If $a_{2}=a_{1}$, this is substituted into equation (4.1a). Solving the corresponding boundary value problem we arrive at

$$
T(r) \sim \frac{1}{2 \varepsilon a_{1}} \int_{r}^{1} \frac{1}{s} \log \left(\frac{a_{1}}{a_{0}} s^{2}+1\right) \mathrm{d} s .
$$

\section{The first special case: angles of deterministic and stochastic load are related in a particular way}

We assume $k_{0}=0$ while $k_{1} \neq 0$. In this case $a_{0}=0, a_{1}>0$. The angles $\varphi_{d}, \varphi_{s}$ are related by

$$
0=k_{1} P_{d}^{*} \sin \varphi_{d}+\sin \varphi_{s}
$$

An important case is the vertically loaded pendulum $\left(\varphi_{d}=\varphi_{s}=0\right)$. Since $a_{0}=0$, the behaviour is qualitatively different from that in Section 4. At $r=0$ we now have a natural boundary, meaning that $r=0$ cannot be reached from the interior of the unit interval, and the interior of the unit interval cannot be reached from $r=0$, in finite time. Let $u_{s}(r)$ now be defined as the probability of exit at $r=1$, starting from a point $r$ of the half open interval $(0,1]$. This probability is obtained by solving the stationary backward equation (3.5a) on the interval $\delta \leqslant r \leqslant 1$ with $0<\delta \ll 1$, where the boundary conditions are $u_{s}^{\prime \prime}(\delta)=0, u_{s}^{\prime \prime}(1)=1$. In the limit $\delta \rightarrow 0$ we obtain

$$
u_{s}(r) \sim\left\{\begin{array}{lll}
1, & a_{2}>a_{1}, & \text { (damping below level } \left.a_{2}=a_{1}\right), \\
r^{1-a_{2} / a_{1}}, & a_{2}<a_{1}, & \text { (damping beyond level } \left.a_{2}=a_{1}\right) .
\end{array}\right.
$$

In the first case of (5.2), exit at $r=1$ occurs with probability one, as in Section 4 the rod is stochastically unstable. In the second case of (5.2), the probability of exit at $r=1$ can be made arbitrarily small by starting close enough to $r=0$, the rod is stochastically stable. We will continue with the stochastically unstable case here; the treatment of the stochastically stable case is postponed until Subsection 5.4. The expected exit time is obtained by solving equation (4.1a) with $a_{0}=0$ under the conditions $\tilde{T}^{0}(1)=0, \tilde{T}^{0}(\delta)=0,0<\delta \ll 1$. In the limit $\delta \rightarrow 0$ :

$$
T(r) \sim \frac{1}{\varepsilon\left(a_{2}-a_{1}\right)} \log \frac{1}{r}, \quad\left(a_{2}>a_{1}\right) .
$$


Because $a_{0}=0$, the differential equations describing the exit problem have a simple form, so that a variety of interesting expressions can be derived.

\subsection{The probability of exit before a certain time $\left(a_{2}>a_{1}\right)$}

Henceforth, the dimensionless time $t^{*}$ will be denoted by $t$, for convenience. Let $u(r, t)$ be the probability that exit through $r=1$ takes place on the time interval $[0, t]$, starting in $r \in(0,1]$ on $t=0$. With $a_{0}=0$ the backward equation (3.5a) reads

$$
\frac{\partial u^{0}}{\partial \tilde{t}}=a_{2} r \frac{\partial u^{0}}{\partial r}+a_{1} r^{2} \frac{\partial^{2} u^{0}}{\partial r^{2}}
$$

This equation has to be solved with the conditions

$$
\begin{aligned}
& u^{0}(r, 0)=0, \\
& u^{0}(\delta, \tilde{t})=0, \quad \delta \rightarrow 0, \\
& u^{0}(1, \tilde{t})=1
\end{aligned}
$$

Taking the Laplace transform with respect to $\tilde{t}$,

$$
\bar{u}^{0}(r, s)=\int_{0}^{\infty} \mathrm{e}^{-s \tilde{t}} u^{\prime \prime}(r, \tilde{t}) \mathrm{d} \tilde{t}
$$

the following boundary value problem is obtained:

$$
\begin{aligned}
& a_{1} r^{2} \frac{\partial^{2} \bar{u}^{-0}}{\partial r^{2}}+a_{2} r \frac{\partial \bar{u}^{0}}{\partial r}=s \bar{u}^{0}-u^{0}(r, 0)=s \bar{u}^{0}, \\
& \bar{u}^{-0}(\delta, s)=0, \quad \delta \rightarrow 0, \\
& \bar{u}^{-0}(1, s)=\frac{1}{s} .
\end{aligned}
$$

The equation (5.6a) is an equidimensional or Euler equation [1], that by the change of variable

$$
r=\mathrm{e}^{\rho}
$$

transforms into

$$
a_{1} \frac{\partial^{2} \bar{u}^{0}}{\partial \rho^{2}}+\left(a_{2}-a_{1}\right) \frac{\partial \bar{u}^{0}}{\partial \rho}-s \bar{u}^{-0}=0,
$$

where the coefficients of the derivatives have become constants. The characteristic equation

$$
a_{1} k^{2}+\left(a_{2}-a_{1}\right) k-s=0
$$


corresponding to this equation is solved by

$$
\begin{aligned}
& k_{1}(s)=\frac{-\left(a_{2}-a_{1}\right)+\sqrt{\left(a_{2}-a_{1}\right)^{2}+4 a_{1} s}}{2 a_{1}}, \\
& k_{2}(s)=\frac{-\left(a_{2}-a_{1}\right)-\sqrt{\left(a_{2}-a_{1}\right)^{2}+4 a_{1} s}}{2 a_{1}} .
\end{aligned}
$$

The solution of $(5.8)$ is

$$
\bar{u}^{0}=C_{1}(s) \mathrm{e}^{k_{1}(s) \rho}+C_{2}(s) \mathrm{e}^{k_{2}(s) \rho},
$$

and that of $(5.6 \mathrm{a})$ :

$$
\bar{u}^{0}=C_{1}(s) r^{k_{1}(s)}+C_{2}(s) r^{k_{2}(s)} .
$$

The unknown functions $C_{1}$ and $C_{2}$ follow from the boundary conditions $(5.6 \mathrm{~b}, \mathrm{c})$,

$$
\lim _{\delta \rightarrow 0} C_{2}(s)=\lim _{\delta \rightarrow 0} \frac{-1}{s\left(\delta^{k_{2}-k_{1}}-1\right)}=0,
$$

in which we used the positivity of $a_{1}$, and, in the same limit:

$$
C_{1}(s)=1 / s
$$

The solution of $(5.6)$ is

$$
\bar{u}^{(0)}(r, s)=r^{k_{1}(s)} / s .
$$

Using some elementary properties of Laplace transforms and the inverse transform formula $5.129[8$, p. 264], we obtain as solution of problem $(5.4)$ :

$$
u^{\prime \prime}\left(r, \frac{\tilde{t}}{a_{1}}\right)=\frac{1}{2} \operatorname{erfc}\left[\frac{\log \frac{1}{r}}{2 \sqrt{\tilde{t}}}-c \sqrt{\tilde{t}}\right]+\frac{1}{2} r^{-2 c} \operatorname{erfc}\left[\frac{\log \frac{1}{r}}{2 \sqrt{\tilde{t}}}+c \sqrt{\tilde{t}}\right],
$$

where

$$
c=\frac{a_{2}-a_{1}}{2 a_{1}}, \quad(0<c \leqslant 1, c=1: \text { no damping }) .
$$

With $u(r, t) \sim u^{0}(r, \tilde{t})$ we obtain an approximation of $u(r, t)$. It is easily verified that this result is in agreement with

$$
\lim _{t \rightarrow \infty} u(r, t)=u_{s}(r)
$$

with $u_{s}(r)=1$, as in the first case of (5.2), where $u_{s}(r)$ was the probability that exit at $r=1$ will occur, starting at $r \in(0,1]$. The function $u$ has been depicted in Fig. 2a. The result 


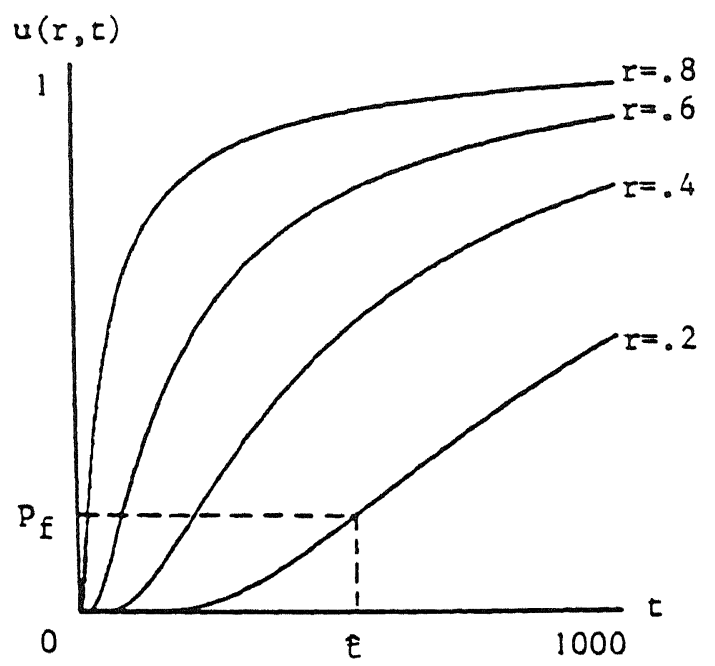

Fig. 2a. The probability $u(r, t)$ for the undamped rod with $\varepsilon=0.01$ and $a_{1}=1 / 16$. For $r=0.2$ the probability of failure exit $p_{f}$ corresponding to an operating time $\hat{t}$ has been indicated.

derived in this subsection differs from formula (3.28) in Katz and Schuss [7], since we took into account the right-hand boundary condition.

As an application, we consider the following problem. What is the probability of failure of the mechanical system during an operating time $\hat{t}$ ? Let $v_{0}(r)$ be the probability density function of the initial $r$ (recall that $r$ is the square root of the dimensionless energy). The probability of failure $p_{f}$ is determined by

$$
p_{f}=\int_{0}^{1} v_{0}(r) u(r, \hat{t}) \mathrm{d} r
$$

In Fig. 2a, the probability of failure for $v_{0}(r)=\delta(r-0.2)$ has been indicated.

\subsection{The exit-time density, its moments and cumulants $\left(a_{2}>a_{1}\right)$}

We define $\tau(r, t) \mathrm{d} t$ as the probability that the time of exit through $r=1$ is in the time interval $(t, t+\mathrm{d} t)$, given that we started at $r \in(0,1]$ on $t=0$. The function $\tau(r, t)$ is a probability density with respect to $t$. In this subsection, we will derive expressions for $\tau(r, t)$ and its $n$-th moment $\mu_{n}(r)$ and cumulant $\kappa_{n}(r)$. The function

$$
1-\int_{0}^{t} \tau\left(r, t^{\prime}\right) \mathrm{d} t^{\prime}
$$

is known to satisfy the backward equation [4]. By differentiation with respect to $t$, it follows that $\tau$ satisfies the backward equation as well, so that for its leading-order term $\tau^{0}$ we have

$$
\frac{\partial \tau^{0}}{\partial \tilde{t}}=a_{1} r^{2} \frac{\partial^{2} \tau^{0}}{\partial r^{2}}+a_{2} r \frac{\partial \tau^{0}}{\partial r}
$$

Taking the Laplace transform of $\tau^{0}$ with respect to $\tilde{t}$,

$$
\bar{\tau}^{0}(r, s)=\int_{0}^{\infty} \mathrm{e}^{-s \tilde{t}} \tau^{0}(r, \tilde{t}) \mathrm{d} \tilde{t}
$$


equation $(5.19)$ becomes

$$
a_{1} r^{2} \frac{\partial^{2} \tau^{\prime \prime}}{\partial r^{2}}+a_{2} r \frac{\partial \tau^{\prime \prime}}{\partial r}=s \tau^{-1}-\tau^{\prime \prime}(r, 0)=s \tau^{\prime \prime},
$$

in which the initial condition

$$
\tau^{0}(r, 0)=0
$$

has been used, valid for $\delta<r<1$, where again $0<\delta \ll 1$. For $r=\delta$ and $r=1$ the probability density function $\tau(r, t)$ equals the delta function $\delta(t-0)$, or

$$
\mu_{0}^{\prime \prime}(r)=1, \quad \mu_{m}^{\prime \prime}(r)=0, \quad m>0 .
$$

where $\mu_{m}^{\prime \prime}(r)$ is the $m$-th moment about the origin $\tilde{t}=0$ of $\varepsilon^{-1} \tau^{\prime \prime}(r, \tilde{t})$. Expression (5.23a) results from the normalisation of the density $\tau(r, t)$ and the expansion

$$
\tau(r, t) \sim \tau^{\prime \prime}(r, \tilde{t})
$$

By the relation $[6, p .6]$,

$$
\varepsilon^{-1} \bar{\tau}^{(1)}(r, s)=G^{\prime \prime}(r, i s)=\sum_{m=0}^{\infty} \frac{(-s)^{m}}{m !} \mu_{m}^{\prime \prime}(r),
$$

in which $G^{0}$ is the characteristic or moment-generating function of $\varepsilon^{-1} \tau^{\prime \prime}$, the conditions (5.23) translate into boundary conditions for (5.21):

$$
\bar{\tau}^{(1)}(1, s)=\bar{\tau}^{(1)}(\delta, s)=\varepsilon, \quad(\delta \rightarrow 0) .
$$

The boundary-value problem $(5.21,5.26)$ is solved in a way similar to that in the previous section. We find

$$
\lim _{\delta \rightarrow 0} C_{2}(s)=\lim _{\delta \rightarrow 0} \varepsilon \frac{\delta^{-k_{1}}-1}{\delta^{k_{2}-k_{1}}-1}=0,
$$

because $a_{1}>0$, and, in this limit,

$$
C_{1}(s)=\varepsilon,
$$

so that

$$
\bar{\tau}^{(1)}(r, s)=\varepsilon r^{k_{1}(s)}
$$

Using the inverse Laplace transform formula 5.85 [8, p. 258], the following result is obtained:

$$
\frac{1}{\varepsilon a_{1}} \tau^{\prime \prime}\left(r, \frac{\tilde{t}}{a_{1}}\right)=\frac{r^{-c}}{2 \sqrt{\pi}}\left(\log \frac{1}{r}\right) \tilde{t}^{-3 / 2} \mathrm{e}^{-\left(-2 \tilde{t}-(\log r)^{2} /(4 \tilde{i})\right.} .
$$

With (5.24), an approximation to $\tau(r, t)$ follows, see Fig. $2 b$. 
$\tau(r, t) / \varepsilon$

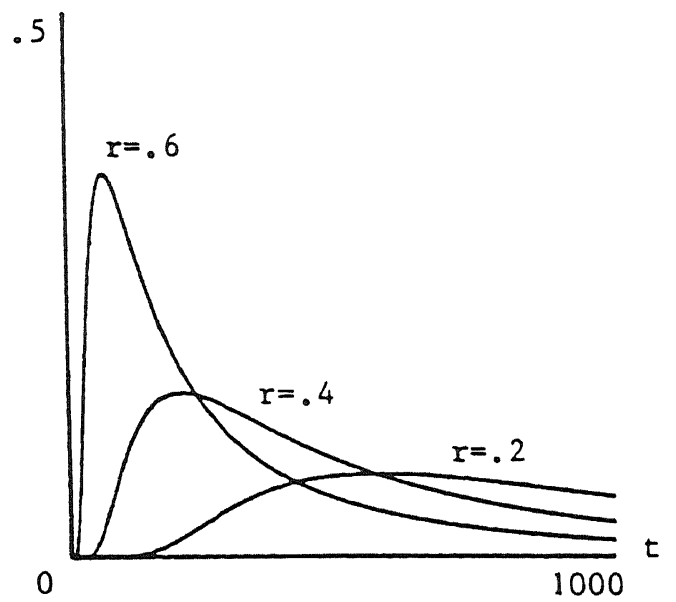

Fig. $2 b$. The probability density function of the exit time, depending on the starting point $r$, for the undamped rod with $\varepsilon=0.01$ and $a_{1}=1 / 16$.

Next we will derive expressions for the moments and cumulants of the density $\tau(r, t)$. The moments $\mu_{n}(r)$ about $t=0$ of $\tau(r, t)$ are found starting from the definition

$$
\mu_{n}(r)=\int_{0}^{\infty} t^{n} \tau(r, t) \mathrm{d} t
$$

Using the obtained approximation of $\tau(r, t)$, and formulas $3.471: 9[5$, p. 340] and 8.468 [5, p. 967], it is found after some calculation that

$$
\mu_{n}(r) \sim\left(4 \varepsilon a_{1} c^{2}\right)^{-n} \sum_{j=1}^{n} \frac{(2 n-j-1) !}{(j-1) !(n-j) !}\left(2 c \log r^{-1}\right)^{j}, \quad n \geqslant 1 .
$$

With (5.28) the moment generating function $G$ of $\tau(r, t)$ yields

$$
G(r, i s)=\bar{\tau}(r, s) \sim \frac{1}{\varepsilon} \bar{\tau}^{(1)}(r, s / \varepsilon)=r^{k_{1}(s / \varepsilon)} .
$$

Taylor expanding the logarithm of $G(r, i s)$ around $s=0$ we find

$$
\log G(r, i s) \sim \sum_{n=1}^{\infty} \frac{(-s)^{n}}{n !} \kappa_{n}(r),
$$

with the cumulants $\kappa_{n}(r)$ given by

$$
\kappa_{n}(r) \sim \frac{(2 n-3) ! !}{\left(2 a_{1} \varepsilon\right)^{n} c^{2 n-1}} \log \frac{1}{r}, \quad n \geqslant 1
$$

the double exclamation mark being defined as in $[5$, p. xliii].

In this subsection we have obtained expressions for the lifetime density of a mechanical system, as well as its moments and cumulants, as functions of the initial $r$. When the initial $r$ is not known, but its density $v_{0}(r)$ is, these expressions should be integrated with respect to $r$ 
from 0 to 1 with the weight function $v_{0}(r)$, as at the end of the previous subsection. This remark applies also to other results in this paper, in particular to the expressions of the expectation of the exit time.

\subsection{Solution of the forward equation $\left(a_{2}>a_{1}\right)$}

Let us define $v(r, t) \mathrm{d} r$ as the probability of being in the interval $(r, r+\mathrm{d} r)$ at time $t$, given the probability $v_{0}\left(r^{\prime}\right) \mathrm{d} r^{\prime}$ of being in the interval $\left(r^{\prime}, r^{\prime}+\mathrm{d} r^{\prime}\right)$ at time $t=0$, where $r$, $r^{\prime} \in(0,1]$. In this subsection, an expression for $v(r, t)$ will be derived. The Fokker-Planck or forward Kolmogorov equation associated with (5.4a) reads

$$
\frac{\partial v^{0}}{\partial \tilde{t}}=-a_{2} \frac{\partial}{\partial r}\left(r v^{0}\right)+a_{1} \frac{\partial^{2}}{\partial r^{2}}\left(r^{2} v^{0}\right)
$$

which has to be solved with the conditions

$$
\begin{aligned}
& v^{0}(r, 0)=v_{0}(r) \\
& v^{0}(\delta, \tilde{t})=0, \quad \delta \rightarrow 0, \\
& v^{0}(1, \tilde{t})=0
\end{aligned}
$$

The last two conditions result from erecting absorbing boundaries at $r=\delta$ and $r=1$, and $v_{0}$ in (5.36a) is the initial density. Equation (5.35) is rewritten as

$$
\frac{\partial v^{0}}{\partial \tilde{t}}=a_{1} r^{2} \frac{\partial^{2} v^{0}}{\partial r^{2}}+\left(4 a_{1}-a_{2}\right) r \frac{\partial v^{0}}{\partial r}+\left(2 a_{1}-a_{2}\right) v^{0} .
$$

Taking the Laplace transform of $v$ with respect to $\tilde{t}$,

$$
\bar{v}^{0}(r, s)=\int_{0}^{\infty} \mathrm{e}^{-s \tilde{t}} v^{0}(r, \tilde{t}) \mathrm{d} \tilde{t}
$$

$(5.36,5.37)$ changes into the boundary-value problem

$$
\begin{aligned}
& a_{1} r^{2} \frac{\partial^{2} \bar{v}^{-1}}{\partial r^{2}}+\left(4 a_{1}-a_{2}\right) r \frac{\partial \bar{v}^{-0}}{\partial r}+\left(2 a_{1}-a_{2}-s\right) \bar{v}^{-0}=-v_{0}(r), \\
& \bar{v}^{-0}(\delta, s)=0, \quad \delta \rightarrow 0 ; \quad \bar{v}^{-0}(1, s)=0 .
\end{aligned}
$$

The change of variable (5.7) turns equation (5.39a) into the equation

$$
a_{1} \frac{\partial^{2} \bar{v}^{-0}}{\partial \rho^{2}}+\left(3 a_{1}-a_{2}\right) \frac{\partial v^{-0}}{\partial \rho}+\left(2 a_{1}-a_{2}-s\right) \bar{v}^{0}=-v_{0}\left(\mathrm{e}^{\rho}\right)
$$

By solving its characteristic equation, the homogeneous equation associated with (5.40) is found to have the independent solutions

$$
\mathrm{e}^{\left(-1-k_{1}(s)\right) \rho}, \quad \mathrm{e}^{\left(-1-k_{2}(s)\right) \rho},
$$


with $k_{1}$ and $k_{2}$ defined in $(5.10)$, and Wronskian

$$
W(\rho)=\left(k_{1}-k_{2}\right) \mathrm{e}^{\left(-k_{1}-k_{2}-2\right) \rho} .
$$

The inhomogeneous equation (5.40) is solved by the method of variation of parameters [1]. In the original variable $r$ its solution reads

$$
\begin{aligned}
\bar{v}^{-1}(r, s) & =\frac{r^{-1-k_{1}}}{a_{1}\left(k_{1}-k_{2}\right)}\left[C_{1}(s)+\int_{\delta}^{r} v_{0}\left(r^{\prime}\right)\left(r^{\prime}\right)^{k_{1}} \mathrm{~d} r^{\prime}\right] \\
& +\frac{r^{-1-k_{2}}}{a_{1}\left(k_{1}-k_{2}\right)}\left[C_{2}(s)+\int_{r}^{1} v_{0}\left(r^{\prime}\right)\left(r^{\prime}\right)^{k_{2}} \mathrm{~d} r^{\prime}\right],
\end{aligned}
$$

in which it is assumed that the initial density $v_{0}$ is sufficiently regular so that the integrands remain finite in the integration domain (note that $k_{1}>0, k_{2}<0$ ). The functions $C_{1}$ and $C_{2}$ are determined by the boundary conditions $(5.39 \mathrm{~b}, \mathrm{c})$. For $C_{2}$ we find

$$
C_{2}(s)=\frac{\delta^{-k_{1}} \int_{\delta}^{1} v_{0}\left(r^{\prime}\right)\left(r^{\prime}\right)^{k_{1}} \mathrm{~d} r^{\prime}-\delta^{-k_{2}} \int_{\delta}^{1} v_{0}\left(r^{\prime}\right)\left(r^{\prime}\right)^{k_{2}} \mathrm{~d} r^{\prime}}{\delta^{-k_{2}}-\delta^{-k_{1}}}
$$

The second term in the nominator is bounded by $v_{0}(\delta)$. This bound is finite if $v_{0}$ is bounded. Then, consequently, in the limit for $\delta \rightarrow 0$,

$$
\begin{aligned}
& C_{2}(s)=-\int_{0}^{1} v_{0}\left(r^{\prime}\right)\left(r^{\prime}\right)^{k_{1}} \mathrm{~d} r^{\prime}, \\
& C_{1}(s)=0 .
\end{aligned}
$$

The solution of (5.39) can be written in the following form:

$$
\bar{v}^{0}(r, s)=\frac{1}{a_{1}\left(k_{1}-k_{2}\right)} \int_{0}^{1} \frac{v_{0}\left(r^{\prime}\right)}{r}\left(\frac{r^{\prime}}{r}\right)^{\left(k_{1}+k_{2}\right) / 2}\left[\mathrm{e}^{-\left|\log r / r^{\prime}\right|\left(k_{1}-k_{2}\right) / 2}-\mathrm{e}^{-\left(\log 1 / r^{\prime} r\right)\left(k_{1}-k_{2}\right) / 2}\right] \mathrm{d} r^{\prime}
$$

The solution of problem $(5.35,5.36)$ is obtained using the inverse Laplace transform 5.87 [ 8 , p. 258]:

$$
v^{0}\left(r, \frac{\tilde{t}}{a_{1}}\right)=\int_{0}^{1} v_{0}\left(r^{\prime}\right) \frac{1}{r}\left(\frac{r}{r^{\prime}}\right)^{c} \frac{\mathrm{e}^{-c^{2} \tilde{t}}}{2 \sqrt{\pi \tilde{t}}}\left[\mathrm{e}^{-\left(\log \frac{r}{r^{\prime}}\right)^{2} /(4 \tilde{t})}-\mathrm{e}^{-\left(\log r^{\prime} r\right)^{2 / 4} \tilde{t}}\right] \mathrm{d} r^{\prime},
$$

and with $v(r, t) \sim v^{0}(r, \tilde{t})$ we have an approximation of $v(r, t)$, see Fig. 2c. The results $(5.15$, $5.29,5.47)$ are related by

$$
\begin{aligned}
& \frac{\partial}{\partial \tilde{t}} u^{0}\left(r, \frac{\tilde{t}}{a_{1}}\right)=\frac{1}{\varepsilon a_{1}} \tau^{0}\left(r, \frac{\tilde{t}}{a_{1}}\right), \\
& 1-\int_{0}^{1} v^{0}\left(r, \frac{\tilde{t}}{a_{1}}\right) \mathrm{d} r=u^{0}\left(r^{*}, \frac{\tilde{t}}{a_{1}}\right), \quad \text { for } v_{0}(r)=\delta\left(r-r^{*}\right) .
\end{aligned}
$$




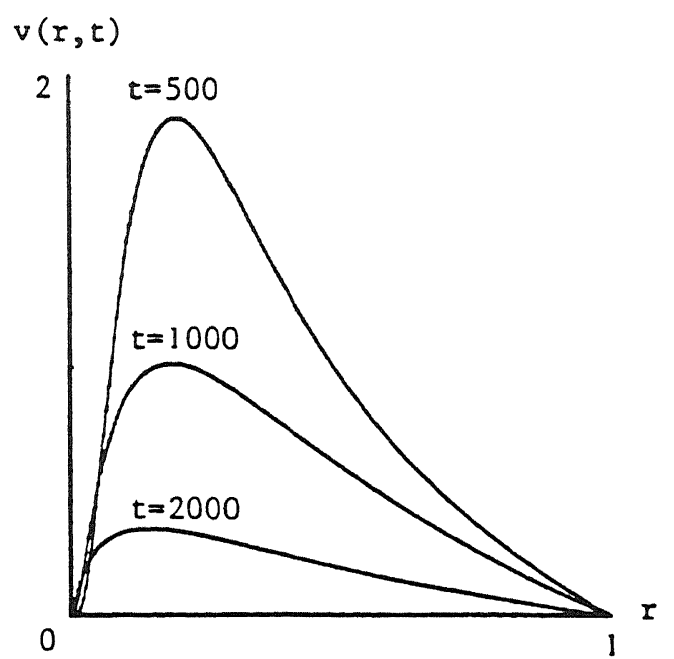

Fig. 2c. The function $v(r, t)$ corresponding to the initial density $v_{01}(r)=\delta(r-0.2)$, for the undamped rod with $\varepsilon=0.01$ and $a_{1}=1 / 16$.

The example in Fig. 2c shows the decay of $v$ with time due to exit at $r=1$. For small $r$ we see an increase of $v$ with time because of systems that are driven to $r=0$, but cannot exit there since $r=0$ is a natural boundary.

As an application of the result derived here, we discuss the construction of confidence intervals. Suppose we are interested in the confidence interval of probability $p$ at time $\hat{t}$, for systems not yet exited (the probability that the system has not yet exited is given by the integral of $v(r, \hat{t})$ with respect to $r$ from 0 to 1$)$. Let $r_{1}$ and $r_{2}$ be the lower and upper boundary of the confidence interval, respectively, so that $0<r_{1}<r_{2}<1$. The values of $r_{1}$ and $r_{2}$ are determined by the conditions

$$
\begin{aligned}
& v\left(r_{1}, \hat{t}\right)=v\left(r_{2}, \hat{t}\right), \\
& \int_{r_{1}}^{r_{2}} v(r, \hat{t}) \mathrm{d} r / \int_{0}^{1} v(r, \hat{t}) \mathrm{d} r=p,
\end{aligned}
$$

from which they can be computed numerically.

\subsection{The stochastically stable case $\left(a_{2}<a_{1}\right)$}

As we have seen at the beginning of Section 5, for stochastically stable systems the probability of exit at $r=1$ is given by

$$
u_{s}^{\prime \prime}(r)=r^{1-a_{2} / a_{1}}
$$

Let $T(r)$ be defined here as the expected time of exit through $r=1$, where it is given that exit through $r=1$ will occur. An expression for $T$ is found by solving the boundary value problem [4, p. 142]:

$$
-u_{s}^{(\prime)}=a_{2} r \frac{\partial}{\partial r}\left(u_{s}^{(1)} \tilde{T}^{()}\right)+a_{1} r^{2} \frac{\partial^{2}}{\partial r^{2}}\left(u_{s}^{(1)} \tilde{T}^{(1)}\right) \text {, }
$$




$$
u_{s}^{\prime \prime}(\delta) \tilde{T}^{0}(\delta)=0, \quad \delta \rightarrow 0 ; \quad u_{s}^{\prime}(1) \tilde{T}^{\prime \prime}(1)=0
$$

We find

$$
T(r) \sim \frac{1}{\varepsilon\left(a_{1}-a_{2}\right)} \log \frac{1}{r} .
$$

Starting at a given position $r$, with increasing damping (decreasing $a_{2}$ ) the probability of exit at $r=1$ decreases according to (5.50), and the mean time of exit at $r=1$, given that exit occurs, decreases as well, according to (5.52). Thus, in systems with damping far beyond the level $a_{2}=a_{1}$ (i.e. $a_{2} \ll a_{1}$ ), starting away from $r=1$, exit is not likely to occur, and if it occurs, it probably is relatively shortly after starting. In such systems, failure mechanisms of a type, different from that discussed in this paper, are of importance. See the remark in Section 8.

\section{The second special case: horizontal stochastic load}

We assume $k_{1}=0$. It follows that $a_{1}=0$. This case corresponds to a horizontal stochastic loading. Using (2.13) we find that $k_{0}^{2}=1$. The backward equation similar to (3.5) reads

$$
\frac{\partial u^{0}}{\partial \tilde{t}}=\left(\frac{a_{0}}{r}+a_{2} r\right) \frac{\partial u^{0}}{\partial r}+a_{0} \frac{\partial^{2} u^{0}}{\partial r^{2}},
$$

with

$$
a_{0}=\frac{1}{8}, \quad a_{2}=-\frac{1}{2} k
$$

This is the backward equation of a Rayleigh process $[4,12]$. As in Section $4, r=0$ is an entrance boundary, $r=1$ is an absorbing boundary, and it can be verified that the probability of exit at $r=1$, starting at any point of the closed unit interval, equals one. For the mean exit time we find

$$
T(r) \sim \frac{1}{\varepsilon a_{2}} \int_{r}^{1}\left[1-\mathrm{e}^{-\left(a_{2} / 2 a_{0}\right) s^{2}}\right] \frac{1}{s} \mathrm{~d} s, \quad\left(a_{2} \neq 0\right) .
$$

\subsection{The subcase of no damping}

In the special case that there is no damping, i.e. $k=0$ and thus $a_{2}=0$, equation (6.1a) becomes

$$
\frac{\partial u^{0}}{\partial \tilde{t}}=a_{0}\left(\frac{1}{r} \frac{\partial u^{0}}{\partial r}+\frac{\partial^{2} u^{0}}{\partial r^{2}}\right) .
$$

For the leading-order term $u^{0}$ in the expansion of $u(r, t)$, defined as in Section 5 , this equation is supplemented with the conditions

$$
u^{0}(r, 0)=0, \quad u^{0}(0, \tilde{t}) \text { is finite, } \quad u^{0}(1, \tilde{t})=1 .
$$


The initial-boundary value problem (6.3) is the same as for axisymmetric heat conduction in a cylinder, which has the solution [11, p. 175]

$$
u^{0}(r, \tilde{t})=1-2 \sum_{i} \frac{J_{0}\left(\xi_{i} r\right) \mathrm{e}^{-a_{0} \xi_{i}^{2} \tilde{t}}}{\xi_{i} J_{1}\left(\xi_{i}\right)},
$$

where the summation extends over the positive roots of

$$
J_{0}\left(\xi_{i}\right)=0
$$

$J_{i}$ being the Bessel function of the first kind of order $i$. The exit-time density yields

$$
\varepsilon^{-1} \tau^{\prime \prime}(r, \tilde{t})=\frac{\partial}{\partial \tilde{t}} u^{\prime \prime}(r, \tilde{t})=2 a_{0} \sum_{i} \frac{\xi_{i} J_{0}\left(\xi_{i} r\right) \mathrm{e}^{-a_{0} \xi_{i}^{2} \tilde{t}}}{J_{1}\left(\xi_{i}\right)}
$$

Next we compute the characteristic function and the first few cumulants corresponding to this density. The differential equation for $\tau^{0}$ yields

$$
\frac{\partial \tau^{0}}{\partial \tilde{t}}=a_{0}\left(\frac{1}{r} \frac{\partial \tau^{0}}{\partial r}+\frac{\partial^{2} \tau^{0}}{\partial r^{2}}\right)
$$

Taking the Laplace transform of $\tau^{0}$ with respect to $\tilde{t}$ we obtain

$$
\frac{\partial^{2} \bar{\tau}^{0}}{\partial r^{2}}+\frac{1}{r} \frac{\partial \bar{\tau}^{0}}{\partial r}-\frac{s}{a_{0}} \bar{\tau}^{0}=0
$$

where we used the initial condition

$$
\tau^{0}(r, 0)=0
$$

The general solution of $(6.8)$ is

$$
\bar{\tau}^{0}(r, s)=c_{1} I_{0}\left(\sqrt{s / a_{0}} r\right)+c_{2} K_{0}\left(\sqrt{s / a_{0}} r\right)
$$

where $I_{0}, K_{0}$ are modified Bessel functions of the first and second kind, respectively. Using the boundary conditions

$$
\bar{\tau}^{0}(1, s)=\varepsilon, \quad \bar{\tau}^{-1}(0, s) \text { is finite }
$$

we find

$$
\bar{\tau}^{0}(r, s)=\varepsilon I_{0}\left(\sqrt{s / a_{0}} r\right) / I_{0}\left(\sqrt{s / a_{0}}\right) .
$$

The characteristic function of $\tau(r, t)$ is given by

$$
G(r, i s) \sim \varepsilon^{-1} \bar{\tau}^{-1}(r, s / \varepsilon) .
$$

The cumulants $\kappa_{n}(r)$ of $\tau(r, t)$ are generated by 


$$
\log G(r, i s) \sim \sum_{n=1}^{\infty} \frac{(-s)^{n}}{n !} \kappa_{n}(r) .
$$

Using the Taylor expansion [5, p. 961]

$$
I_{0}(s)=\sum_{k=0}^{\infty} \frac{\left(\frac{s}{2}\right)^{2 k}}{(k !)^{2}}
$$

the cumulants are obtained as

$$
\kappa_{n}(r) \sim \frac{\beta_{n}}{\left(\varepsilon a_{0}\right)^{n}}\left(1-r^{2 n}\right),
$$

where the $\beta_{n}$ are found from the recurrent relations

$$
\sum_{i=1}^{n} \beta_{i}(-4)^{i}(i !) i\left(\begin{array}{c}
n \\
i
\end{array}\right)^{2}=-n, \quad(n=1,2,3 \ldots) .
$$

The first few $\beta$ 's are given by

$$
\beta_{1}=\frac{1}{4}, \quad \beta_{2}=\frac{1}{32}, \quad \beta_{3}=\frac{1}{96} .
$$

\section{Examples in dimensional variables}

Below we give some of the results in dimensional form. Exclusively for the present section, let $T$ denote the dimensional exit time and $r, R$ the dimensional energy and dimensional critical energy, respectively. Using (2.11), (2.13) we find in the case of the undamped rod $\left(a_{2}=3 a_{1}\right)$, according to formulas (4.2), (5.3) and (6.16) respectively, that for the regular case:

$$
T(r) \sim \frac{8 \gamma \tilde{m} \tilde{\mu} l^{2}}{P_{s}^{2}} \log \sqrt{\frac{\tilde{\mu} R^{2} \cos ^{2} \varphi_{s}+2\left(P_{d} \sin \varphi_{d} \cos \varphi_{s}+l \tilde{\mu} \sin \varphi_{s}\right)^{2}}{\tilde{\mu} r^{2} \cos ^{2} \varphi_{s}+2\left(P_{d} \sin \varphi_{d} \cos \varphi_{s}+l \tilde{\mu} \sin \varphi_{s}\right)^{2}}},
$$

for the vertically loaded rod:

$$
T(r) \sim \frac{8 \gamma \tilde{m} \tilde{\mu} l^{2}}{P_{s}^{2}} \log \frac{R}{r}
$$

and for a horizontal stochastic loading:

$$
T(r) \sim \frac{\left(R^{2}-r^{2}\right) 2 \gamma \tilde{m}}{P_{s}^{2}}
$$

with $\tilde{m}, \tilde{\mu}$ defined in (2.8). Recall that in order for these formulas to be applicable, the dimensionless parameter combinations in (2.13) must have the required order of magnitude. This is especially important for the last parameter group in (2.13). If the damping constant is 
large, so that this group is larger than indicated, for example of order 1 , then the exit time is exponentially large, see $[10$, Ch. 7.4] or [4, p. 362], and the model presently described clearly does not apply.

\section{Discussion}

In this paper we have studied the exit problem on the time scale of order $O\left(\varepsilon^{-1}\right)$. We have distinguished three cases, depending on the values of $k_{0}$ and $k_{1}$. The case treated in Section 4 has been called regular, in the sense that an arbitrary choice of $k_{0}$ and $k_{1}$ (or $\varphi_{d}$ and $\varphi_{s}$ ) will almost always lead to this case. In Sections 5 and 6 the special cases $k_{0}=0$ and $k_{1}=0$ have been treated, respectively. Confining ourselves to the first special case (a similar remark applies to the second special case) it can be remarked that in practice $k_{0}$, will never be equal to zero exactly. For example, due to practical imperfections, an exactly vertically loaded rod does not exist. In view of the asymptotics that we have used, the left side of (5.1) may be replaced by $O\left(\varepsilon^{1 / 2}\right)$ in order for the first special case to remain applicable. As an example, consider a nearly vertically loaded rod with $a_{2}>a_{1}$. If relation (5.1) is satisfied within $\mathrm{O}\left(\varepsilon^{1 / 2}\right)$, the mean exit time is given by (5.3), otherwise it is given by (4.2) with small $a_{0}$ measuring the deviation from exactly vertical. For the undamped rod $\left(a_{2}=3 a_{1}\right)$ these exit times yield respectively:

$$
\begin{aligned}
& T(r) \sim \frac{1}{2 \varepsilon a_{1}} \log \frac{1}{r}, \\
& T(r) \sim \frac{1}{2 \varepsilon a_{1}} \log \sqrt{\frac{a_{1}+a_{0}}{a_{1} r^{2}+a_{0}}},
\end{aligned}
$$

which have been depicted in Fig. 3 for $a_{0}=0.01$ and $a_{1}=1, r$ being the square root of the dimensionless energy. Moreover, we see that (8.1a) is a good approximation to (8.1b) away from $r=0$.

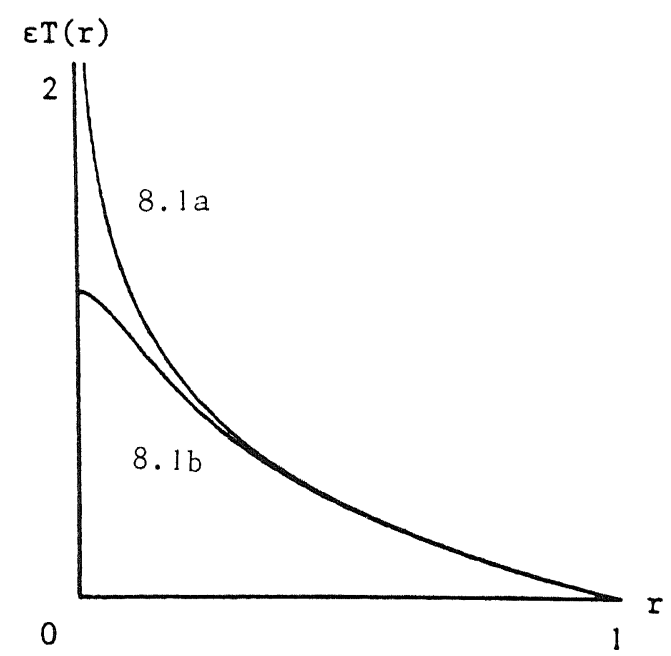

Fig. 3. The expected exit time for the nearly vertically loaded undamped rod according to the formulas (8.1a) and (8.1b). 
The expressions (4.2), (4.3), (5.3), (6.2), and (6.16) with $n=1$, give the expected time $T(r)$ needed in the various cases considered to reach a critical energy level (which, in these cases, will happen with probability one). This critical energy was chosen as the upper bound of the normal operating energy range of a system. Given a probability density function $v_{0}(r)$ of the initial $r$, we can adopt the constant

$$
T_{f}=\int_{0}^{1} v_{0}(r) T(r) \mathrm{d} r
$$

as the expected failure time for that system.

The formulas (5.34), (6.16) with $n=2$ (higher cumulants are often of less practical interest) can be used to indicate the variance in failure time. It was not possible to obtain similar simple expressions for all cases considered. In such cases the variance is easily obtained numerically by solving a recurrent system of elliptic boundary-value problems [4, p. 138 and p. 171].

Due to the simplicity of the equations in some special cases, we were able to find expressions for the probability density of the exit time, see (5.29) and (6.6). In practice the failure density is obtained by fitting experimental data with some statistical density (exponential, gamma, Weibull or lognormal), see for example [9]. In the present paper we showed how to derive such a density by employing the dynamics of the system.

A stock or investment policy can be based on the expression for $u$ obtained in Section 5.1. Given an operating time $\hat{t}$ and a probability density of initial energies, the probability of failure is determined by (5.17). The expression for $v$ in Section 5.3 can be used for the construction of confidence intervals.

The stochastic stability of oscillators with a different type of damping (as cubic damping) or noise (red, dichotomic, etc.), and with a forcing that can be described by a potential function, has been treated by Dygas, Matkowsky and Schuss [2].

Finally, it may be remarked that in practice failure mechanisms, different from the type considered here, may be present. One such a mechanism, to mention, is wear out, which becomes important, especially in systems with damping beyond the level $a_{2}=a_{1}$. An approach to wear out could be as follows. Assume that wear out depends on the state $r \in[0,1]$ and the time $t \in(0, \infty)$, expressed for some application by a penalty function $P(r, t)$. Let $S(r, t) \mathrm{d} r$ be the expected time spent in $(r, r+\mathrm{d} r)$ during the time interval $(0, t)$,

$$
S(r, t)=\int_{0}^{t} v\left(r, t^{\prime}\right) \mathrm{d} t^{\prime}
$$

where $v(r, t) \mathrm{d} r$ is the probability of being in $(r, r+\mathrm{d} r)$ on time $t$ (i.e. the solution of the forward equation). A wear-out function can be defined by

$$
W(t)=\int_{0}^{1} P(r, t) S(r, t) \mathrm{d} r .
$$

The failure time $t_{f}$ follows from

$$
W\left(t_{f}\right)=W_{c},
$$

where $W_{c}$ is a critical value, so that excess of this value leads to failure of the system. In 
general, the computations involved become very complicated, so that in practice this scheme has to be simplified somewhere.

\section{Acknowledgements}

I am grateful to Johan Grasman for the discussions concerning the subject treated in this paper and to Nico Temme for some remarks.

\section{References}

1. C.M. Bender and S.A. Orszag, Advanced mathematical methods for scientists and engineers, McGraw-Hill Book Company (1978).

2. M.M. Dygas, B.J. Matkowsky and Z. Schuss, Stochastic stability of nonlinear oscillators, Technical Report No. 8615, Appl. Math. Northwestern University, Evanston, Illinois (1987).

3. W. Feller, The parabolic differential equations and the associated semigroups of transformations, Ann. of Math. 55 (1952) 468-519.

4. C.W. Gardiner, Handbook of stochastic methods, for physics, chemistry and the natural sciences, Springer-Verlag (1983).

5. I.S. Gradshteyn and I.M. Ryzhik, Table of integrals, series, and products; corrected and enlarged edition, Academic Press (1980).

6. N.G. van Kampen, Stochastic processes in physics and chemistry, North-Holland Publishing Company (1981)

7. A. Katz and Z. Schuss, Reliability of elastic structures driven by random loads, SIAM J. Appl. Math. 45 (1985) 383-402.

8. F. Oberhettinger and L. Badii, Tables of Laplace transforms, Springer-Verlag (1973).

9. C.P. Quesenberry and J. Kent, Selecting among probability distributions used in reliability, Technometrics 24 (1982) 59-65.

10. Z. Schuss, Theory and applications of stochastic differential equations, Wiley (1980).

11. I.N. Sneddon, Fourier transforms, McGraw-Hill Book Company (1951).

12. R.L. Stratonovich, Topics in the theory of random noise, Vol. 1, Gordon and Breach (1963). 